\title{
Orbital Targeting Using Reduced Eccentric Anomaly Low-Thrust Coefficients
}

\author{
Jennifer S. Hudson* \\ University of Michigan, Ann Arbor, Michigan 48019 \\ and \\ Daniel J. Scheeres \\ University of Colorado, Boulder, Colorado 80309 \\ DOI: $\underline{10.2514 / 1.51336}$
}

\begin{abstract}
A method to evaluate the trajectory dynamics of low-thrust spacecraft is applied to targeting and optimal control problems. Averaged variational equations for the osculating orbital elements are used to estimate a spacecraft trajectory over many spiral orbits. Fourteen Fourier coefficients of the thrust acceleration vector represent the fundamental trajectory dynamics. Spacecraft targeting problems are solved using the averaged variational equations and a general cost function represented as a Fourier series. The resulting fuel costs and dynamic fidelity of the targeting solutions are evaluated. The goal of the method is not precise targeting, but easy reconstruction of the basic elements of the thrusting trajectory and control law.
\end{abstract}

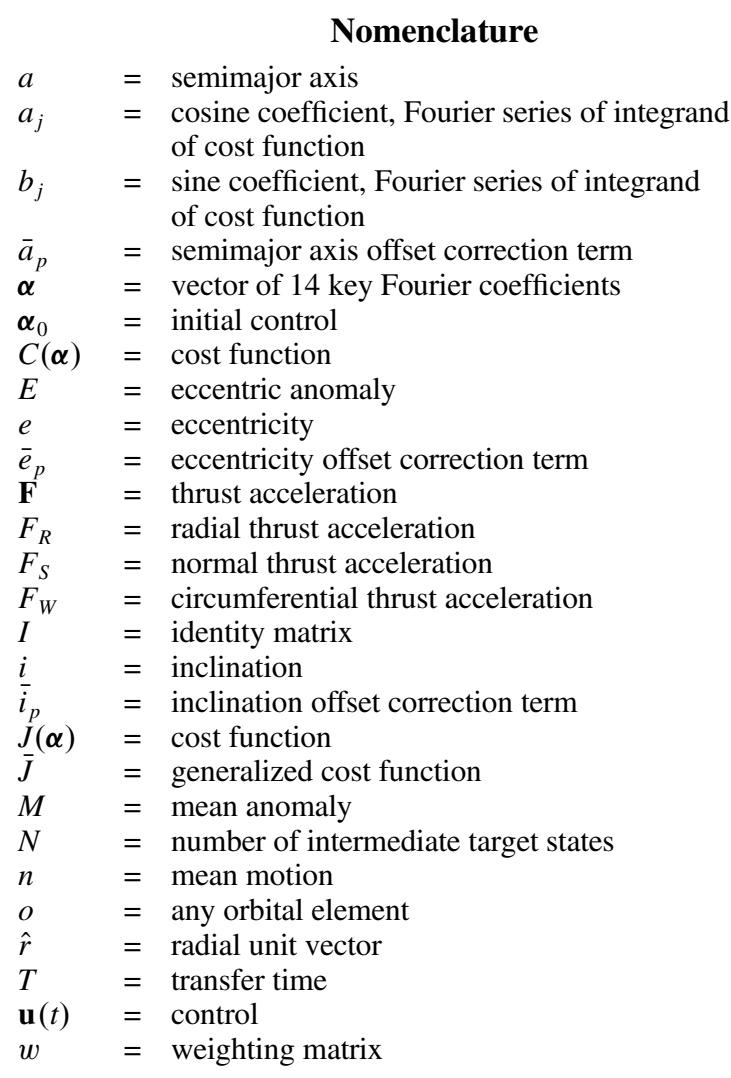

Presented at the 20TH AAS/AIAA Space Flight Mechanics Meeting, San Diego, CA, 14-17 February 2010; received 25 June 2010; revision received 7 January 2011; accepted for publication 10 January 2011. Copyright @ 2011 by Jennifer Hudson. Published by the American Institute of Aeronautics and Astronautics, Inc., with permission. Copies of this paper may be made for personal or internal use, on condition that the copier pay the $\$ 10.00$ per-copy fee to the Copyright Clearance Center, Inc., 222 Rosewood Drive, Danvers, MA 01923; include the code 0731-5090/11 and $\$ 10.00$ in correspondence with the CCC.

*Ph.D. Candidate, Department of Aerospace Engineering, 1320 Beal Ave.; jshudson@umich.edu.

${ }^{\dagger}$ A. Richard Seebass Professor, Department of Aerospace Engineering Sciences, Colorado Center for Astrodynamics Research. Associate Fellow AIAA.

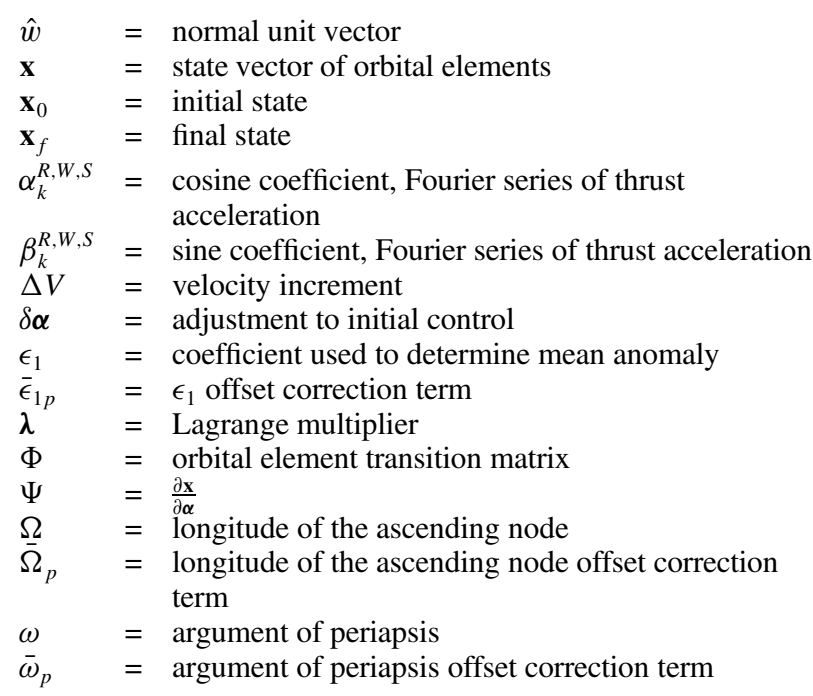

\section{Introduction}

A DVANCES in electric propulsion have made low-thrust $A$ engines a growing trend in the spacecraft industry over the past few decades. The Deep Space 1, SMART-1, Hayabusa, Dawn, and GOCE missions have demonstrated this technology, and it is slated for launch on the LISA Pathfinder and BepiColombo missions, among others. With high specific impulse and long engine lifetimes, low-thrust propulsion is well-suited for many applications, including orbit transfers with many spiral orbits.

Optimal control of low-thrust spacecraft, however, remains a difficult problem. The general continuous-thrust problem requires integration of the Newtonian equations of motion for the full trajectory, which may comprise hundreds of orbits and is highly sensitive to small changes in the thrust profile. Analytical solutions exist for several special cases of low-thrust orbit transfer problems, such as Forbes's spiral [1], the logarithmic spiral [1-3], the exponential sinusoid [4], the case of constant radial or circumferential thrust [5-7], the case of variable radial thrust [8-10], Markopoulos' Keplerian thrust arcs [11], Lawden's spiral [1ㄹ] , and Bishop and Azimov's spiral [13]. The calculus of variations [14] and direct optimization methods [15] have also been used to determine optimal low-thrust control laws within certain constraints. Several methods for open-loop, minimum-time transfers [16-18] and 
optimal transfers using Lyapunov feedback control $[\underline{19}, \underline{20}]$ also exist. Averaging methods, in combination with other approaches, have proven effective in overcoming sensitivities to small variations in initial orbit and thrust profile [21-24]. Yet all of these solutions remain limited to certain regions of the thrust and orbital parameter space.

Previous work by Hudson and Scheeres [25] has established the validity of an averaging method to model the trajectory dynamics of low-thrust spacecraft without these parameter restrictions. The spacecraft thrust vector components are represented as Fourier series in eccentric anomaly and the Gauss variational equations are averaged over one orbit to define a set of secular equations. The secular equations are functions of only 14 of the thrust Fourier coefficients, regardless of the order of the original Fourier series, and can represent thrust controls of varying magnitude and direction.

This method considers the three orthogonal components of the thrust acceleration vector separately. The thrust in each direction may be variable, constant, or zero. There are no upper or lower bounds on the thrust, except that it must be of sufficiently low magnitude that the size and shape of the orbit do not change significantly over one revolution.

This paper describes the application of this method to orbital targeting problems. Using a two-body Newtonian model, targeting problems ranging from five to approximately 80 revolutions are solved without restrictions on the orbital elements (except that eccentricity may not be zero) or thrust direction. General two-point boundary value problems are solved using an iterative method that converges on the minimum-norm set of the 14 force Fourier coefficients. Problems involving sequences of target states are also solved, either by finding the optimal set of control coefficients between each pair of states, or by fitting a single set of control coefficients to the entire orbit transfer using a least-squares approach. The resulting fuel costs and dynamic fidelity of the targeting solutions are evaluated. The goal of the targeting method is not precision, but easy reconstruction of the basic elements of the thrusting trajectory and control law. These methods require significantly reduced computing resources compared with integration of the full Newtonian equations of motion.

Trajectory analysis using the reduced Fourier coefficients has several potential fields of application, including mission design and space situational awareness (SSA). Mission designers could use the averaged secular equations to efficiently estimate control laws for a large number of potential orbital paths, to compare fuel costs and other trajectory characteristics, and to estimate the feasibility and cost of proposed deviations from a selected path. In SSA applications, observers could reconstruct the orbital path of a suspected low-thrust spacecraft from a few discrete observations to identify the fundamental characteristics of the control law used, estimate fuel consumption, and predict the future trajectory. The different methods developed for solving orbital targeting problems and calculating equivalent control laws have different strengths and weaknesses that make them appropriate, respectively, for these different applications.

\section{Methods}

Previously, Hudson and Scheeres [25] developed a method to model average low-thrust trajectory dynamics using 14 fundamental coefficients. This section is intended to summarize the elements of that method that are relevant to orbital targeting. For further details and validating examples, readers are referred to [25].

Secular equations can be derived by averaging the Gauss form of the Lagrange Planetary Equations over one orbit period with respect to mean anomaly. The acceleration vector is assumed to have a sufficiently low magnitude that the size and shape of the orbit do not change significantly over one revolution

$$
\overline{\dot{o}}=\frac{1}{2 \pi} \int_{0}^{2 \pi} \dot{o} \mathrm{~d} M
$$

Here $o$ represents any orbit element. The thrust acceleration vector $\mathbf{F}$ is resolved along the radial, normal, and circumferential directions.
Each component of the thrust acceleration is represented as a Fourier series expanded in eccentric anomaly

$$
\begin{gathered}
\mathbf{F}=F_{R} \hat{\mathbf{r}}+F_{W} \hat{\mathbf{w}}+F_{S}(\hat{\mathbf{w}} \times \hat{\mathbf{r}}) \\
F_{R}=\sum_{k=0}^{\infty}\left[\alpha_{k}^{R} \cos k E+\beta_{k}^{R} \sin k E\right] \\
F_{W}=\sum_{k=0}^{\infty}\left[\alpha_{k}^{W} \cos k E+\beta_{k}^{W} \sin k E\right] \\
F_{S}=\sum_{k=0}^{\infty}\left[\alpha_{k}^{S} \cos k E+\beta_{k}^{S} \sin k E\right]
\end{gathered}
$$

where $\hat{\mathbf{r}}=\frac{\mathbf{r}}{|\mathbf{r}|}$ and $\hat{\mathbf{w}}=\frac{\mathbf{r} \times \mathbf{v}}{|\mathbf{r} \times \mathbf{v}|}$. When these Fourier series for the thrust vector components are substituted into the averaged Gauss equations and the independent parameter for the averaging is shifted to eccentric anomaly, the orthogonality conditions eliminate all but the zeroth-, first-, and second-order coefficients of each thrust acceleration Fourier series. Thus, the averaged secular Gauss equations are:

$$
\begin{aligned}
& \overline{\dot{a}}=2 \sqrt{\frac{a^{3}}{\mu}}\left[\frac{1}{2} e \beta_{1}^{R}+\sqrt{1-e^{2}} \alpha_{0}^{S}\right] \\
& \overline{\dot{e}}=\sqrt{\frac{a}{\mu}} \sqrt{1-e^{2}}\left[\frac{1}{2} \sqrt{1-e^{2}} \beta_{1}^{R}+\alpha_{1}^{S}-\frac{3}{2} e \alpha_{0}^{S}-\frac{1}{4} e \alpha_{2}^{S}\right] \\
& \bar{i}=\sqrt{\frac{a}{\mu}} \frac{1}{\sqrt{1-e^{2}}}\left[\frac{1}{2}\left(1+e^{2}\right) \cos \omega \alpha_{1}^{W}-\frac{3}{2} e \cos \omega \alpha_{0}^{W}\right. \\
& \left.-\frac{1}{2} \sqrt{1-e^{2}} \sin \omega \beta_{1}^{W}-\frac{1}{4} e \cos \omega \alpha_{2}^{W}+\frac{1}{4} e \sqrt{1-e^{2}} \sin \omega \beta_{2}^{W}\right] \\
& \overline{\dot{\Omega}}=\sqrt{\frac{a}{\mu}} \frac{\csc i}{\sqrt{1-e^{2}}}\left[\frac{1}{2} \sqrt{1-e^{2}} \cos \omega \beta_{1}^{W}+\frac{1}{2}\left(1+e^{2}\right) \sin \omega \alpha_{1}^{W}\right. \\
& \left.-\frac{3}{2} e \sin \omega \alpha_{0}^{W}-\frac{1}{4} e \sqrt{1-e^{2}} \cos \omega \beta_{2}^{W}-\frac{1}{4} e \sin \omega \alpha_{2}^{W}\right] \\
& \overline{\dot{\omega}}=\sqrt{\frac{a}{\mu}} \frac{1}{e}\left[-\frac{1}{2} \sqrt{1-e^{2}} \alpha_{1}^{R}+e \sqrt{1-e^{2}} \alpha_{0}^{R}+\frac{1}{2}\left(2-e^{2}\right) \beta_{1}^{S}\right. \\
& \left.-\frac{1}{4} e \beta_{2}^{S}\right]-\cos i \overline{\dot{\Omega}} \\
& \overline{\dot{\epsilon}}_{1}=\sqrt{\frac{a}{\mu}}\left[\left(-2-e^{2}\right) \alpha_{0}^{R}+2 e \alpha_{1}^{R}-\frac{1}{2} e^{2} \alpha_{2}^{R}\right] \\
& +\left(1-\sqrt{1-e^{2}}\right)(\overline{\dot{\omega}}+\overline{\dot{\Omega}})+2 \sqrt{1-e^{2}} \sin ^{2}\left(\frac{i}{2}\right) \overline{\dot{\Omega}}
\end{aligned}
$$

The element $\epsilon_{1}$ is used to determine the mean anomaly, 


$$
M=\int n \mathrm{~d} t+\epsilon_{1}-(\Omega+\omega)
$$

Average trajectories calculated using Eqs. (ㅁ-11) show the correct trends in the evolution of the osculating orbital elements and are good approximations of the true trajectories. However, they may be offset from the true averages and may diverge from true trajectories over many orbits. This may be partially due to higher order effects not captured in the averaging method, but it is often due to nontrivial periodic components which can shift the mean value of the state from the initial condition. This initial condition offset can be corrected by the addition of an averaged periodic term to the initial conditions of the secular equation for each orbital element. These correction terms, $\bar{a}_{p}, \bar{e}_{p}, \bar{i}_{p}, \bar{\Omega}_{p}, \bar{\omega}_{p}, \bar{\epsilon}_{1 p}$, are given in [25].

In [25], the averaged secular equations with corrected initial conditions were shown to efficiently and accurately evaluate the trajectory dynamics of low-thrust spacecraft. Numerical examples demonstrated close agreement between the Newtonian equations of motion and these averaged secular equations over many orbits of general, low-thrust trajectories.

The method has certain limits of applicability. The averaged secular equations are developed only for the restricted two-body model; other gravitational perturbations or spacecraft mass effects must be considered separately. The thrust acceleration must be able to be represented by a Fourier series, as is true for almost any physical system. The resulting controls are periodic in eccentric anomaly, unless the control Fourier coefficients are changed from orbit to orbit. The Gauss equations are singular for circular orbits; however, this method can be used for all orbits with nonzero eccentricity. This method can also model coast arcs by setting the thrust Fourier series to zero.

\section{Targeting}

The averaged secular variational equations provide an efficient means of solving low-thrust orbit transfer problems. Any spacecraft targeting problem with a time span sufficient to allow low-thrust transfer can theoretically be solved by numerical integration of the averaged equations and iterative evaluation of the 14 thrust Fourier coefficients.

Orbital targeting problems with fixed endpoint constraints are considered. These problems take the form of two-point boundary value problems in which a spacecraft must transfer from an initial state to a final state, each defined by six orbital elements, in a fixed amount of time.

The averaged equations solve these problems for the secular orbital elements. The solutions will not precisely agree with the true spacecraft trajectory, as calculated by the Newtonian equations of motion, due to the short period offsets between them. However, the secular solutions are useful approximations, as they represent the average of the full solution. They may be used to estimate flight requirements, such as velocity increment and fuel consumption, or to initialize other optimization methods to determine the true optimal trajectory.

\section{A. Two-Point Boundary Value Problems}

The targeting problem in the averaged equations is approached as a two-point boundary value problem with given initial state $\mathbf{x}_{0}$, final state $\mathbf{x}_{f}$, and transfer time $T$. The equations of motion have the form

$$
\dot{\mathbf{x}}=G(\mathbf{x}) \boldsymbol{\alpha}+F(\mathbf{x})
$$

where $\mathbf{x}$ is the state vector of orbital elements, $\boldsymbol{\alpha}$ is the $14 \times 1$ vector of thrust Fourier coefficients that appear in the averaged secular equations, and $F(\mathbf{x})$ accounts for the additional mean motion term in the differential equation for mean anomaly

$$
\mathbf{x}=\left[\begin{array}{c}
a \\
e \\
i \\
\Omega \\
\omega \\
M
\end{array}\right], \quad \boldsymbol{\alpha}=\left[\begin{array}{c}
\alpha_{0}^{R} \\
\alpha_{1}^{R} \\
\alpha_{2}^{R} \\
\beta_{1}^{R} \\
\alpha_{0}^{S} \\
\alpha_{1}^{S} \\
\alpha_{2}^{S} \\
\beta_{1}^{S} \\
\beta_{2}^{S} \\
\alpha_{0}^{W} \\
\alpha_{1}^{W} \\
\alpha_{2}^{W} \\
\beta_{1}^{W} \\
\beta_{2}^{W}
\end{array}\right], \quad \mathbf{F}=\left[\begin{array}{l}
0 \\
0 \\
0 \\
0 \\
0 \\
n
\end{array}\right]
$$

The term $G(\mathbf{x}) \boldsymbol{\alpha}$ represents Eqs. (6-11).

Solutions to these problems will involve only the secular orbital elements. The true trajectory, as calculated by the Newtonian equations, will not match these solutions exactly due to the short period offsets. Nonetheless, these secular solutions should accurately represent the averaged dynamics of the true trajectory and are thus useful for many applications.

An initial control that does not meet the targeting objectives is assumed

$$
\mathbf{x}_{f} \neq \mathbf{x}\left(T, \boldsymbol{\alpha}_{0}, \mathbf{x}_{0}\right)
$$

A small adjustment is added to $\boldsymbol{\alpha}_{0}$ to make the state at time $T$ equal to the final state

$$
\mathbf{x}_{f}=\mathbf{x}\left(T, \boldsymbol{\alpha}_{0}+\delta \boldsymbol{\alpha}, \mathbf{x}_{0}\right)
$$

This is expanded as its first-order Taylor series approximation

$$
\mathbf{x}\left(T, \boldsymbol{\alpha}, \mathbf{x}_{0}\right) \approx \mathbf{x}\left(T, \boldsymbol{\alpha}_{0}, \mathbf{x}_{0}\right)+\frac{\partial \mathbf{x}}{\partial \boldsymbol{\alpha}} \cdot \delta \boldsymbol{\alpha}
$$

where $\boldsymbol{\alpha}=\boldsymbol{\alpha}_{0}+\delta \boldsymbol{\alpha}$, and solved for $\left(\frac{\partial \mathbf{x}}{\partial \boldsymbol{\alpha}}\right) \delta \boldsymbol{\alpha}$

$$
\left(\frac{\partial \mathbf{x}}{\partial \boldsymbol{\alpha}}\right) \delta \boldsymbol{\alpha}=\left(\mathbf{x}_{f}-\mathbf{x}\left(T, \boldsymbol{\alpha}_{0}, \mathbf{x}_{0}\right)\right)
$$

However, this problem has an infinity of possible solutions as there are 14 free variables in $\boldsymbol{\alpha}$ and only 6 constraint equations. Therefore an additional constraint is added, imposing that the one solution that minimizes a cost function $J(\boldsymbol{\alpha})$ must be chosen.

The problem then becomes a constrained minimization problem, which can be solved by defining a generalized cost function that includes the boundary value constraints multiplied by a Lagrange multiplier vector

$$
\bar{J}=J(\boldsymbol{\alpha})+\lambda\left[\mathbf{x}\left(T, \boldsymbol{\alpha}, \mathbf{x}_{0}\right)-\mathbf{x}_{f}\right]
$$

Here the Langrange multiplier $\lambda$ is represented as a six-dimensional row vector. The necessary condition to minimize $J(\alpha)$ and satisfy the boundary value conditions is

$$
\frac{\partial \bar{J}}{\partial(\boldsymbol{\alpha}, \boldsymbol{\lambda})}=[\mathbf{0}]_{20 \times 1}=\left[\begin{array}{c}
\frac{\partial J}{\partial \boldsymbol{\alpha}}+\lambda \frac{\partial \mathbf{x}}{\partial \boldsymbol{\alpha}} \\
\mathbf{x}\left(T, \boldsymbol{\alpha}, \mathbf{x}_{0}\right)-\mathbf{x}_{f}
\end{array}\right]
$$

The final state $\mathbf{x}\left(T, \boldsymbol{\alpha}, \mathbf{x}_{0}\right)$ is again replaced with its Taylor series approximation, Eq. (16). For clarity, let $\frac{\partial \mathbf{x}}{\partial \alpha}=\Psi$. To evaluate this matrix, the partial derivative of Eq. (13) is taken with respect to $\alpha$,

$$
\frac{\partial}{\partial \boldsymbol{\alpha}}(\dot{\mathbf{x}})=\left[\frac{\partial \mathbf{F}}{\partial \mathbf{x}}+\boldsymbol{\alpha} \frac{\partial G}{\partial \mathbf{x}}\right]\left(\frac{\partial \mathbf{x}}{\partial \boldsymbol{\alpha}}\right)+G(\mathbf{x})
$$

The $\boldsymbol{\alpha} \frac{\partial G}{\partial \mathbf{x}}$ term, which will be small compared with $G(\mathbf{x})$ if the perturbing thrust coefficients are small, is ignored for computational convenience. Solutions that use this approach converge, so this neglect is believed to be appropriate. Equation (20) now has the form 


$$
\begin{gathered}
\dot{\Psi}(t)=\frac{\partial \mathbf{F}(x)}{\partial \mathbf{x}} \Psi(t)+G(\mathbf{x}(t)) \\
\Psi(0)=0
\end{gathered}
$$

Note that the matrix $\frac{\partial \mathbf{F}}{\partial \mathbf{x}}$ has only one nonzero element. The homogeneous system,

$$
\begin{gathered}
\dot{\Psi}_{h}=\left[\begin{array}{cccccc}
0 & 0 & 0 & 0 & 0 & 0 \\
0 & 0 & 0 & 0 & 0 & 0 \\
0 & 0 & 0 & 0 & 0 & 0 \\
0 & 0 & 0 & 0 & 0 & 0 \\
0 & 0 & 0 & 0 & 0 & 0 \\
-\frac{3}{2} \frac{n}{a} & 0 & 0 & 0 & 0 & 0
\end{array}\right] \Psi \\
\Psi_{h}(t)=\left[\begin{array}{cccccc}
1 & 0 & 0 & 0 & 0 & 0 \\
0 & 1 & 0 & 0 & 0 & 0 \\
0 & 0 & 1 & 0 & 0 & 0 \\
0 & 0 & 0 & 1 & 0 & 0 \\
0 & 0 & 0 & 0 & 1 & 0 \\
-\frac{3}{2} \frac{n}{a} t & 0 & 0 & 0 & 0 & 1
\end{array}\right] \Psi_{0}=\Phi(t) \Psi_{0} \\
\end{gathered}
$$

is solved, where $\Phi=\frac{\partial \mathbf{x}}{\partial \mathbf{x}_{0}}$ is the orbital element transition matrix.

Using the standard procedure, the particular solution is found, then the homogeneous and particular solutions are summed at $t=0$ to find the general solution,

$$
\Psi(t)=\Phi(t) \int_{0}^{t} \Phi^{-1}(\tau) G(\mathbf{x}(\tau)) \mathrm{d} \tau
$$

This expression for $\Psi$ can be used to numerically find the solutions for the necessary conditions (19). Equation (26) can be integrated concurrently with the trajectory to calculate $\bar{\Psi}$ at each time step. Beginning with the zero thrust case, the thrust coefficient vector $\boldsymbol{\alpha}=\boldsymbol{\alpha}_{0}+\delta \boldsymbol{\alpha}$ is then iteratively updated by solving the two simultaneous vector equations

$$
\begin{gathered}
\left.\frac{\partial J}{\partial \boldsymbol{\alpha}}\right|_{\boldsymbol{\alpha}+\delta \boldsymbol{\alpha}}+\lambda \cdot \Psi=0 \\
\mathbf{x}\left(T, \boldsymbol{\alpha}_{0}, \mathbf{x}_{0}\right)+\Psi \cdot \delta \boldsymbol{\alpha}-\mathbf{x}_{f}=0
\end{gathered}
$$

A cost function of the form

$$
J(\boldsymbol{\alpha})=\frac{1}{2 \pi} \int_{0}^{2 \pi} f(\mathbf{F}) \mathrm{d} M
$$

is assumed, where $\mathbf{F}$ represents the force due only to the 14 relevant terms in the component Fourier series. This total cost is evaluated over one orbit, which agrees with the averaging result. Thus, this is proportional to the average cost of the given thrust law.

If the integrand of the cost function is represented as a Fourier series

$$
f(\mathbf{F})=\sum_{j=0}^{\infty} a_{j} \cos (j E)+b_{j} \sin (j E)
$$

and the independent variable of integration is shifted to eccentric anomaly, only the first two terms of the Fourier series remain:

$$
J(\boldsymbol{\alpha})=\frac{1}{2 \pi} \int_{0}^{2 \pi}\left[\sum_{j=0}^{\infty} a_{j} \cos (j E)+b_{j} \sin (j E)\right](1-e \cos E) \mathrm{d} E
$$

$$
=a_{0}-\frac{e}{2} a_{1}
$$

By the definition of Fourier coefficients

$$
a_{0}=\frac{1}{2 \pi} \int_{0}^{2 \pi} f(\mathbf{F}) \mathrm{d} E
$$

$$
a_{1}=\frac{1}{2 \pi} \int_{0}^{2 \pi} f(\mathbf{F}) \cos (E) \mathrm{d} E
$$

Evaluation of Eq. (27) requires the partial derivative $\frac{\partial J(\alpha)}{\partial \alpha}$, which has the form

$$
\frac{\partial J(\boldsymbol{\alpha})}{\partial \boldsymbol{\alpha}}=\frac{\partial a_{0}}{\partial \boldsymbol{\alpha}}-\frac{e}{2} \frac{\partial a_{1}}{\partial \boldsymbol{\alpha}}
$$

This partial derivative can be evaluated numerically for most cost functions and may be evaluated analytically for certain simple cost functions.

First, consider a cost function $J(\alpha)$ that represents the minimum of the norm of the acceleration vector for the targeting problem, or the total velocity increment of the thrust over one orbit. A negligible rate of mass loss is assumed, such that this cost function is simply the average thrust per orbit

$$
J(\boldsymbol{\alpha})=\frac{1}{2 \pi} \int_{0}^{2 \pi} \sqrt{F_{R}^{2}+F_{S}^{2}+F_{W}^{2}} \mathrm{~d} M
$$

Equations (32) and (33) become

$$
\begin{gathered}
a_{0}=\frac{1}{2 \pi} \int_{0}^{2 \pi} \sqrt{F_{R}^{2}+F_{S}^{2}+F_{W}^{2}} \mathrm{~d} E \\
a_{1}=\frac{1}{2 \pi} \int_{0}^{2 \pi} \sqrt{F_{R}^{2}+F_{S}^{2}+F_{W}^{2}} \cos (E) \mathrm{d} E
\end{gathered}
$$

However, the partial derivatives of these coefficients are difficult to evaluate analytically and are undefined in the zero thrust case

$$
\begin{gathered}
\frac{\partial a_{0}}{\partial \boldsymbol{\alpha}}=\frac{1}{4 \pi} \int_{0}^{2 \pi} \frac{\frac{\partial}{\partial \alpha}\left(F_{R}^{2}+F_{S}^{2}+F_{W}^{2}\right)}{\sqrt{F_{R}^{2}+F_{S}^{2}+F_{W}^{2}}} \mathrm{~d} E \\
\frac{\partial a_{0}}{\partial \boldsymbol{\alpha}}=\frac{1}{4 \pi} \int_{0}^{2 \pi} \frac{\frac{\partial}{\partial \alpha}\left(F_{R}^{2}+F_{S}^{2}+F_{W}^{2}\right) \cos (j E)}{\sqrt{F_{R}^{2}+F_{S}^{2}+F_{W}^{2}}} \mathrm{~d} E
\end{gathered}
$$

To avoid this problem, consider a different cost function $J(\alpha)$, the square of the norm, representing the minimum energy solution

$$
J(\boldsymbol{\alpha})=\frac{1}{2 \pi} \int_{0}^{2 \pi}\left(F_{R}^{2}+F_{S}^{2}+F_{W}^{2}\right) \mathrm{d} M
$$

This cost function provides an upper bound on the velocity increment, $\Delta V$, per unit time. The $\Delta V$ between two time periods, $t_{0}$ and $t_{0}+\delta t$, is

$$
\begin{gathered}
\Delta V=\int_{t_{0}}^{t_{0}+\delta t}\|\mathbf{u}(t)\| \mathrm{d} t \\
=\int_{t_{0}}^{t_{0}+\delta t} \sqrt{F_{R}^{2}+F_{S}^{2}+F_{W}^{2}} \mathrm{~d} t
\end{gathered}
$$


$\int_{t_{0}}^{t_{0}+\delta t} \sqrt{F_{R}^{2}+F_{S}^{2}+F_{W}^{2}} \mathrm{~d} t \leq \sqrt{\int_{t_{0}}^{t_{0}+\delta t}\left(\sqrt{F_{R}^{2}+F_{S}^{2}+F_{W}^{2}}\right)^{2} \mathrm{~d} t \cdot \int_{t_{0}}^{t_{0}+\delta t} \mathrm{~d} t}$

$$
\leq \sqrt{\int_{t_{0}}^{t_{0}+\delta t}\left(F_{R}^{2}+F_{S}^{2}+F_{W}^{2}\right) \mathrm{d} t \cdot \delta t}
$$

Therefore, $\Delta V$ is bounded by

$$
\Delta V \leq \sqrt{2 \delta t} \sqrt{J(\boldsymbol{\alpha})}
$$

The expressions for Eqs. (32) and (33) are found using the expansion and simplification shown in the Appendix

$$
\begin{gathered}
a_{0}=\frac{1}{2}\left(\boldsymbol{\alpha} \cdot \boldsymbol{\alpha}+\left(\alpha_{0}^{R}\right)^{2}+\left(\alpha_{0}^{S}\right)^{2}+\left(\alpha_{0}^{W}\right)^{2}\right) \\
a_{1}=\frac{1}{2}\left(2 \alpha_{0}^{R} \alpha_{1}^{R}+\alpha_{1}^{R} \alpha_{2}^{R}+2 \alpha_{0}^{S} \alpha_{1}^{S}+\alpha_{1}^{S} \alpha_{2}^{S}+\beta_{1}^{S} \beta_{2}^{S}+2 \alpha_{0}^{W} \alpha_{1}^{W}\right. \\
\left.+\alpha_{1}^{W} \alpha_{2}^{W}+\beta_{1}^{W} \beta_{2}^{W}\right)
\end{gathered}
$$

Now the partial derivative $\frac{\partial J(\boldsymbol{\alpha})}{\partial \boldsymbol{\alpha}}$ is straightforward to evaluate analytically:

$$
\frac{\partial J(\boldsymbol{\alpha})}{\partial \boldsymbol{\alpha}}=\boldsymbol{\alpha}\left(Q-\frac{1}{2} e Z\right)
$$

where

$$
\begin{aligned}
& Q=\left[\begin{array}{ccc}
q_{4 \times 4} & & \mathbf{0} \\
\mathbf{0} & q_{5 \times 5} & \\
& & q_{5 \times 5}
\end{array}\right]_{14 \times 14}, \quad q_{4 \times 4}=\left[\begin{array}{cccc}
2 & & & \mathbf{0} \\
& 1 & & \\
& & 1 & \\
\mathbf{0} & & & 1
\end{array}\right] \\
& q_{5 \times 5}=\left[\begin{array}{ccccc}
2 & & & & 0 \\
& 1 & & & \\
& & 1 & & \\
& & & 1 & \\
\mathbf{0} & & & & 1
\end{array}\right] \\
& Z=\left[\begin{array}{ccc}
z_{4 \times 4} & & \mathbf{0} \\
& z_{5 \times 5} & \\
\mathbf{0} & & z_{5 \times 5}
\end{array}\right]_{14 \times 14}, \quad z_{4 \times 4}=\frac{1}{2}\left[\begin{array}{cccc}
0 & 2 & 0 & 0 \\
2 & 0 & 1 & 0 \\
0 & 1 & 0 & 0 \\
0 & 0 & 0 & 0
\end{array}\right] \\
& z_{5 \times 5}=\frac{1}{2}\left[\begin{array}{ccccc}
0 & 2 & 0 & 0 & 0 \\
2 & 0 & 1 & 0 & 0 \\
0 & 1 & 0 & 0 & 0 \\
0 & 0 & 0 & 0 & 1 \\
0 & 0 & 0 & 1 & 0
\end{array}\right]
\end{aligned}
$$

Equations (27) and (28) can now be solved to iteratively update $\boldsymbol{\alpha}$ :

$$
\begin{gathered}
\delta \boldsymbol{\alpha}=-\left(Q-\frac{1}{2} e Z\right)^{-T} \Psi^{T} \lambda^{T}-\boldsymbol{\alpha}_{0} \\
\lambda^{T}=\left[\Psi\left(Q-\frac{1}{2} e Z\right)^{-T} \Psi^{T}\right]^{-1}\left(\mathbf{x}\left(T ; \boldsymbol{\alpha}, \mathbf{x}_{0}\right)-\mathbf{x}_{f}-\Psi \boldsymbol{\alpha}_{0}\right)
\end{gathered}
$$

The initial condition offset corrections described in [25] improve the accuracy of the targeting algorithm. The algorithm is initialized with zero thrust, then the calculated Fourier coefficients are used to
Table 1 2PBVP targeting example: initial and target states

\begin{tabular}{ccc}
\hline \hline & Initial state & Final state \\
\hline$a, \mathrm{~km}$ & 7500 & 7700 \\
$e$ & 0.1 & 0.15 \\
$i, \mathrm{deg}$ & 20 & 25 \\
$\Omega, \mathrm{deg}$ & 20 & 25 \\
$\omega, \mathrm{deg}$ & 20 & 25 \\
$M, \mathrm{deg}$ & 0 & 0 \\
Time, h & 0 & 8.9778 \\
\hline \hline
\end{tabular}

Table 2 2PBVP targeting example: final thrust acceleration Fourier coefficients after nine iterations

\begin{tabular}{lrlrrr}
\hline \hline Radial & & \multicolumn{2}{c}{ Normal } & \multicolumn{2}{c}{ Circumferential } \\
\hline$\alpha_{0}^{R}, \mathrm{~m} / \mathrm{s}^{2}$ & -0.0889 & $\alpha_{0}^{W}, \mathrm{~m} / \mathrm{s}^{2}$ & -0.0038 & $\alpha_{0}^{S}, \mathrm{~m} / \mathrm{s}^{2}$ & 0.0014 \\
$\alpha_{1}^{R}, \mathrm{~m} / \mathrm{s}^{2}$ & -0.0007 & $\alpha_{1}^{W}, \mathrm{~m} / \mathrm{s}^{2}$ & 0.0396 & $\alpha_{1}^{S}, \mathrm{~m} / \mathrm{s}^{2}$ & 0.0025 \\
$\alpha_{2}^{R}, \mathrm{~m} / \mathrm{s}^{2}$ & -0.0007 & $\alpha_{2}^{W}, \mathrm{~m} / \mathrm{s}^{2}$ & 0.0009 & $\alpha_{2}^{S}, \mathrm{~m} / \mathrm{s}^{2}$ & 0.0001 \\
$\beta_{1}^{R}, \mathrm{~m} / \mathrm{s}^{2}$ & 0.0237 & $\beta_{1}^{W}, \mathrm{~m} / \mathrm{s}^{2}$ & -0.0025 & $\beta_{1}^{S}, \mathrm{~m} / \mathrm{s}^{2}$ & 0.0144 \\
& & $\beta_{2}^{W}, \mathrm{~m} / \mathrm{s}^{2}$ & -0.0000 & $\beta_{2}^{S}, \mathrm{~m} / \mathrm{s}^{2}$ & 0.0007 \\
\hline \hline
\end{tabular}

correct the initial state of the subsequent iteration. Thus each integration of the averaged equations has a slightly different initial condition which more closely approximates the average of the desired transfer trajectory. The initial condition of the $j$ th iteration is:

$$
\mathbf{x}_{0}(j)=\left[\begin{array}{c}
a_{0}+\bar{a}_{p}(j-1) \\
e_{0}+\bar{e}_{p}(j-1) \\
i_{0}+\bar{i}_{p}(j-1) \\
\Omega_{0}+\bar{\Omega}_{p}(j-1) \\
\omega_{0}+\bar{\omega}_{p}(j-1) \\
\epsilon_{10}+\bar{\epsilon}_{1 p}(j-1)
\end{array}\right]
$$

This method converges to a set of 14 Fourier coefficients that describes a control law that solves the targeting problem in the averaged secular equations. The true trajectory under this control law, as determined by the Newtonian equations, generally compares well with the averaged trajectory.

An example of this targeting method applied to a transfer in five orbital elements is shown below. Table 1 shows the initial and final states of the boundary value problem, between which the spacecraft must transfer. The targeting algorithm was initialized with the zero thrust case and terminated when the thrust coefficients changed by

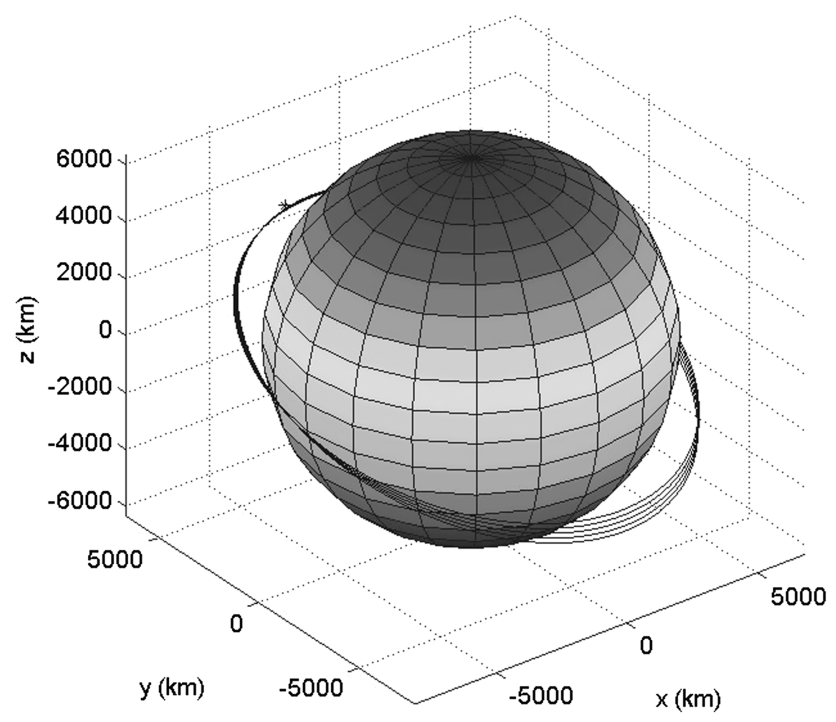

Fig. 1 Two-point boundary value problem (2PBVP) targeting example: solution trajectory after nine iterations. 

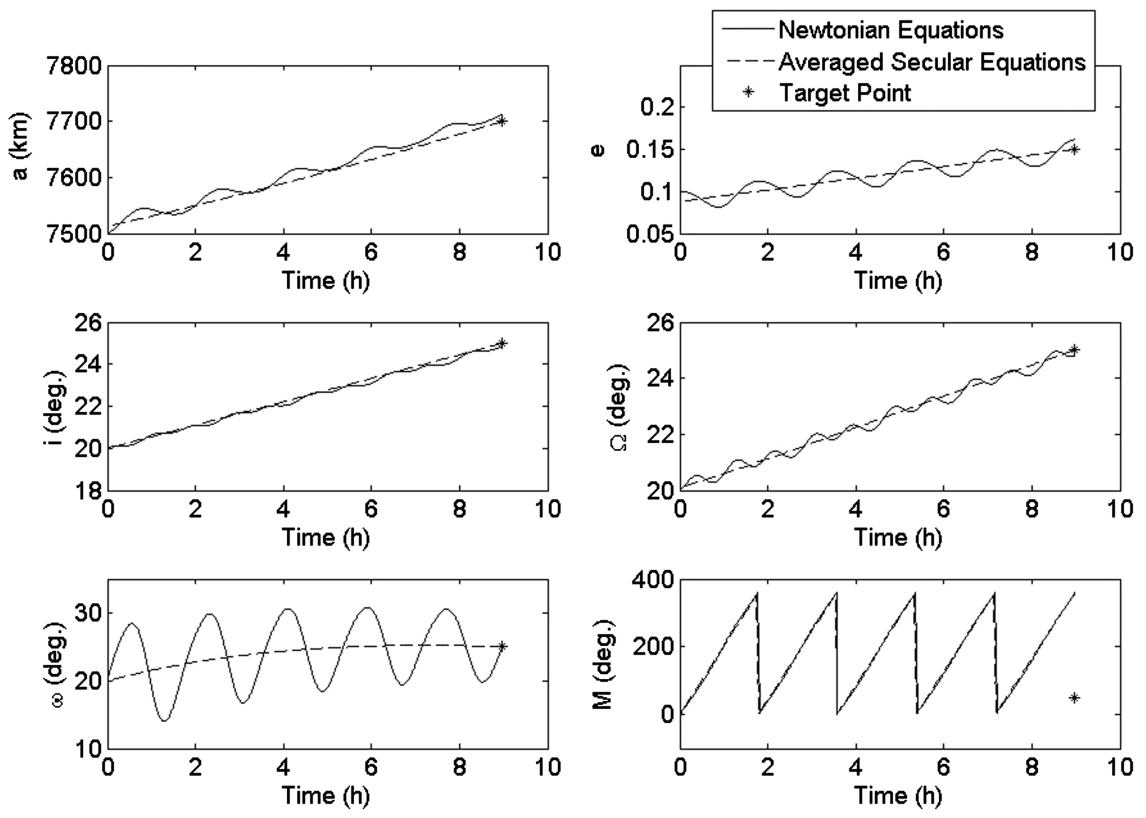

Fig. 2 2PBVP targeting example: solution trajectory after nine iterations.

Table 3 Target States for SMART-1 Example

\begin{tabular}{lccccccc}
\hline \hline & Time, h & $a, \mathrm{~km}$ & $e$ & $i, \mathrm{deg}$ & $\Omega, \mathrm{deg}$ & $\omega, \mathrm{deg}$ & $M, \mathrm{deg}$ \\
\hline $\begin{array}{l}\text { Initial } \\
\text { state }\end{array}$ & 0 & 26433 & 0.671 & 6.915 & 160.315 & 194.821 & 3.136 \\
Target 1 & 155.833 & 26825 & 0.662 & 6.907 & 158.793 & 197.752 & 3.138 \\
Target 2 & 342.702 & 27815 & 0.640 & 6.871 & 157.153 & 200.66 & 3.128 \\
Target 3 & 487.163 & 28713 & 0.622 & 6.861 & 156.084 & 202.442 & 3.142 \\
Target 4 & 680.502 & 29745 & 0.604 & 6.838 & 154.886 & 204.387 & 3.143 \\
Target 5 & 853.156 & 30235 & 0.598 & 6.848 & 153.996 & 205.839 & 3.142 \\
Target 6 & 1017.363 & 31380 & 0.573 & 6.826 & 153.178 & 207.342 & 3.144 \\
\hline \hline
\end{tabular}

less than $0.1 \%$ between iterations. This occurred after nine iterations. The final $\boldsymbol{\alpha}$ is shown in Table $\underline{2}$ and the transfer trajectory is shown in Figs. 1 and 2 . All simulations in this paper were integrated using MATLAAB's ode45 function.

The simple example given by Table 1 is intended to illustrate the differences between the true spacecraft trajectory and the average solution. The true spacecraft trajectory, as calculated by the Newtonian equations of motion, does not match the secular solution exactly due to the short period offsets between them. In solving these two-point boundary value problems, the equations of motion are solved only for the secular orbital elements, so precise agreement with the Newtonian equation solutions is not expected.

This method can also be used to solve more complex targeting problems involving sequences of two or more target states. A lengthy orbit transfer may be approached as a series of two-point boundary value problems and the optimal set of control coefficients may be calculated between each pair of states.

An example of this targeting through a series of states is shown below. The target points, shown in Table 3 , approximately reflect the states of the SMART-1 spacecraft in the early days of its mission. These states occur at fixed times over approximately 80 orbit periods.

Starting with the given initial state and zero thrust, the vector $\boldsymbol{\alpha}$ was iteratively updated to converge on a control law that drove the average trajectory to the first target point. The first target point was then used as the initial condition for the next two-point boundary value problem, and so on. The iterative algorithm for each pair of states was terminated when the thrust coefficients changed by less

${ }^{\ddagger}$ Additional data available at http://sci.esa.int/science-e/www/object/ index.cfm?fobjectid $=31517 \&$ farchive_objecttypeid $=30$ [retrieved December 2010]. than $0.1 \%$ between iterations, which happened after four to six iterations. Figures $\underline{3}$ and $\underline{4}$ show the final trajectory. The velocity increment for this orbit transfer is $1300.6 \mathrm{~m} / \mathrm{s}$.

The average trajectory is continuous throughout the transfer; however, the true trajectory may have discontinuities at the intermediate target states due to short-period variations. Figure $\underline{5}$ is a detail view of the semimajor axis plot near the first target state, in which one of these discontinuities can be seen. In this example, corrections of short-period offsets of the initial conditions, which would also lead to discontinuities in the trajectory, are not included. In practice, these discontinuities would make it impossible to actually implement the trajectory, making this approach inappropriate for many applications.

In some cases, however, calculation of the precise trajectory through the target states is not necessary. In the early stages of mission planning, for example, the target points may represent general mission goals with some flexibility in the actual trajectory. This two-point boundary value method may be appropriate for these applications.

\section{B. Least-Squares Regression}

In the second method for fitting sequences of orbital states, a leastsquares regression approach is used to calculate the low-thrust trajectory. Here, priority is given to finding a single control law for the entire orbit transfer, over precise targeting of the states. The problem is approached using the averaged equations with a given initial state $\mathbf{x}_{0}$, a series of $N$ intermediate target states $\mathbf{x}=\left[x\left(t_{1}\right), x\left(t_{2},\right) \ldots, x\left(t_{N}\right)\right]$, and a set of times associated with each target state $T=\left[t_{1}, t_{2}, \ldots, t_{N}\right]$. The equations of motion again have the form of Eq. (13). To carry this out, a cost function of the form 


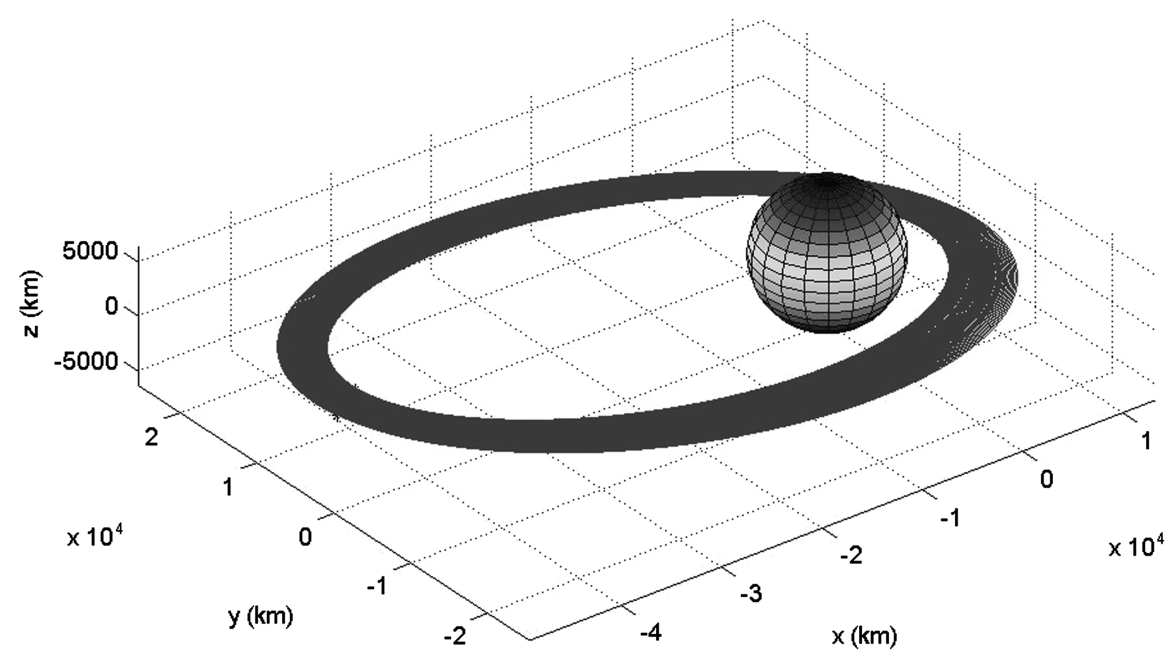

Fig. 3 SMART-1 targeting example: trajectory calculated by 2PBVP method.
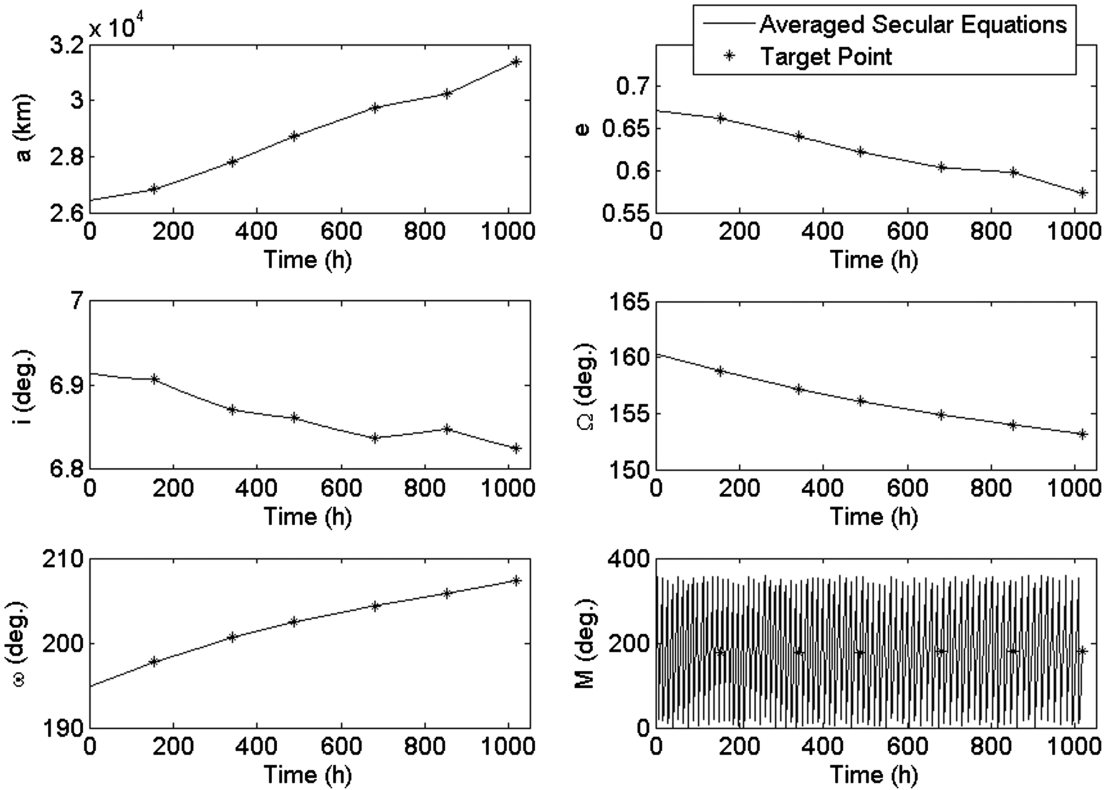

Fig. 4 SMART-1 targeting example: trajectory calculated by 2PBVP method.

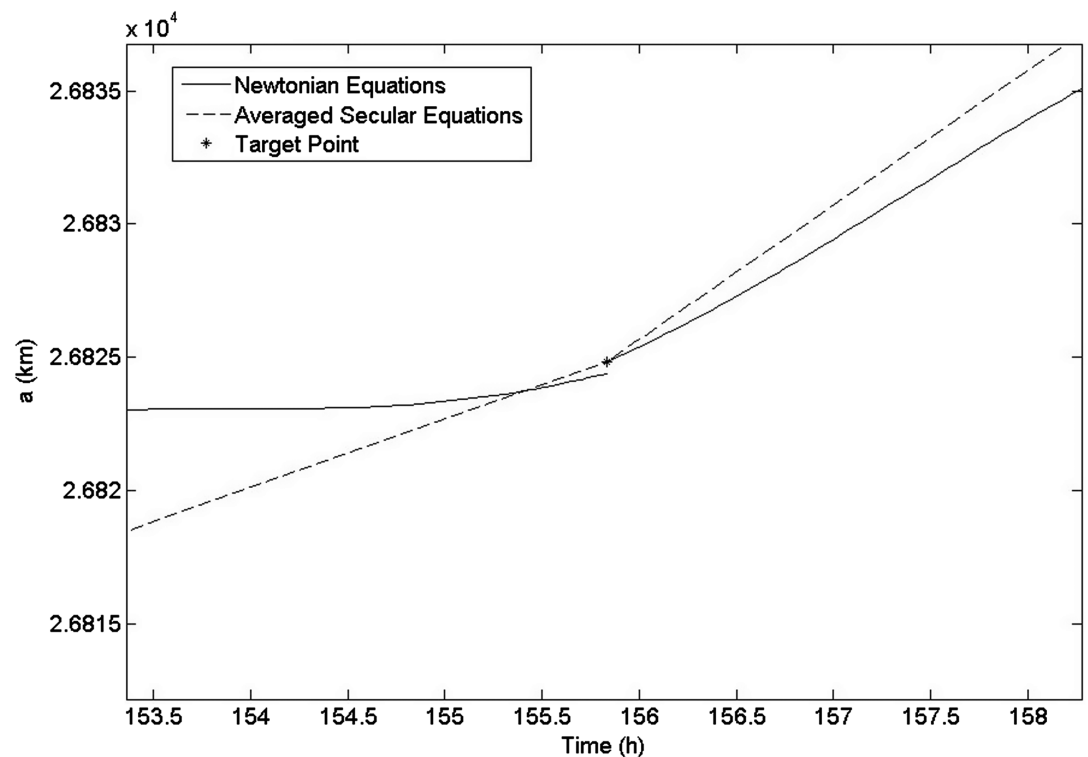

Fig. 5 SMART-1 targeting example: detail of discontinuity near first target state, semimajor axis. 


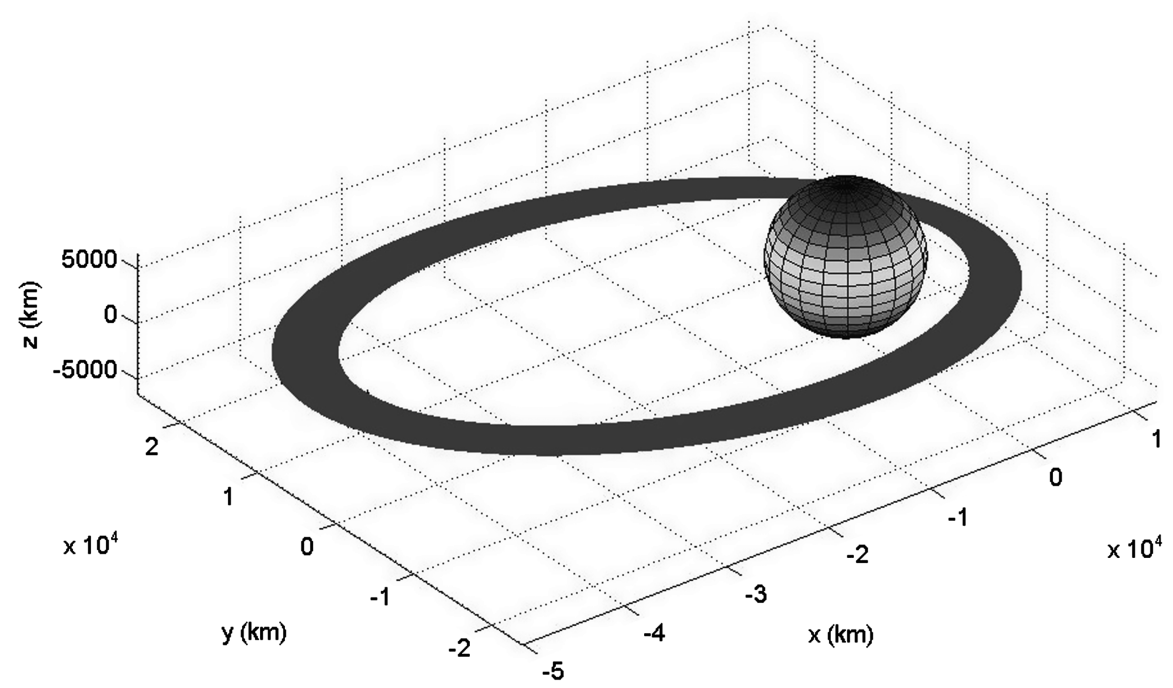

Fig. 6 SMART-1 targeting example: trajectory calculated by least-squares method.

$$
C(\boldsymbol{\alpha})=\frac{1}{2} \sum_{i=1}^{N}\left[\mathbf{x}\left(\boldsymbol{\alpha}, t_{i}\right)-\mathbf{x}_{i}\right] \cdot\left[\mathbf{x}\left(\boldsymbol{\alpha}, t_{i}\right)-\mathbf{x}_{i}\right]
$$

could be used. The necessary condition

$$
\frac{\partial C}{\partial \boldsymbol{\alpha}}=\sum_{i=1}^{N}\left[\mathbf{x}\left(\boldsymbol{\alpha}, t_{i}\right)-\mathbf{x}_{i}\right]\left(\frac{\partial \mathbf{x}}{\partial \boldsymbol{\alpha}}\right)_{i}=0
$$

would be used to minimize $C(\boldsymbol{\alpha})$. The state at each target time, $\mathbf{x}\left(\boldsymbol{\alpha}, t_{i}\right)$, would be replaced with its Taylor series approximation, Eq. (16), and then Eq. (55) would be solved for $\delta \boldsymbol{\alpha}$ to iteratively converge on a solution

$$
\delta \boldsymbol{\alpha}=\left[\sum_{i=1}^{N}\left(\mathbf{x}_{i}-\mathbf{x}\left(\boldsymbol{\alpha}_{0}, t_{i}\right)\right)\left(\frac{\partial \mathbf{x}}{\partial \boldsymbol{\alpha}}\right)_{i}\right]\left[\sum_{i=1}^{N}\left(\frac{\partial \mathbf{x}}{\partial \boldsymbol{\alpha}}\right)_{i}^{T}\left(\frac{\partial \mathbf{x}}{\partial \boldsymbol{\alpha}}\right)_{i}\right]^{-1}
$$

However, the second bracketed term in Eq. (56) is singular in most simulations. This is due to the fact that solutions are not unique, so more than one possible thrust profile may solve the problem. To resolve this issue, another constraint is added, a minimum-energy cost function that must be minimized while solving the least-squares targeting problem

$$
\bar{J}=J(\boldsymbol{\alpha})+\sum_{i=1}^{N}\left[\mathbf{x}\left(\boldsymbol{\alpha}, t_{i}\right)-\mathbf{x}_{i}\right] w\left[\mathbf{x}\left(\boldsymbol{\alpha}, t_{i}\right)-\mathbf{x}_{i}\right]
$$

The minimum-energy cost function $J(\alpha)$, defined by Eq. (40), is minimized while also minimizing the least-squares difference between $\mathbf{x}\left(\boldsymbol{\alpha}, t_{i}\right)$ and $\mathbf{x}_{i}$. A weighting matrix $w$, a $6 \times 6$ matrix of constants that can be independently selected to normalize numerical differences between variables or emphasize certain elements, is introduced. The necessary conditions are

$$
\frac{\partial \bar{J}}{\partial(\boldsymbol{\alpha})}=[\mathbf{0}]_{14 \times 1}=\frac{\partial J}{\partial \boldsymbol{\alpha}}+\sum_{i=1}^{N}\left[\mathbf{x}\left(\boldsymbol{\alpha}, t_{i}\right)-\mathbf{x}_{i}\right] w(\Psi)_{i}
$$

The matrix $\left(\frac{\partial \mathbf{x}}{\partial \alpha}\right)_{i}=(\Psi)_{i}$ is calculated from Eq. (26). The partial derivative for the cost function $J(\alpha)$ is evaluated by Eq. (48).

To solve Eq. $(\underline{58}), \mathbf{x}\left(\boldsymbol{\alpha}, t_{i}\right)$ is replaced with its Taylor series approximation, Eq. (16), and the thrust coefficient vector $\boldsymbol{\alpha}=\boldsymbol{\alpha}_{0}+$ $\delta \boldsymbol{\alpha}$ is iteratively updated

$$
0=\left(\boldsymbol{\alpha}_{0}+\delta \boldsymbol{\alpha}\right)^{T}\left(Q-\frac{1}{2} e Z\right)+\sum_{i=1}^{N}\left[\mathbf{x}\left(\boldsymbol{\alpha}_{0}, t_{i}\right)+\Psi_{i} \delta \boldsymbol{\alpha}-\mathbf{x}_{i}\right] w \Psi_{i}
$$
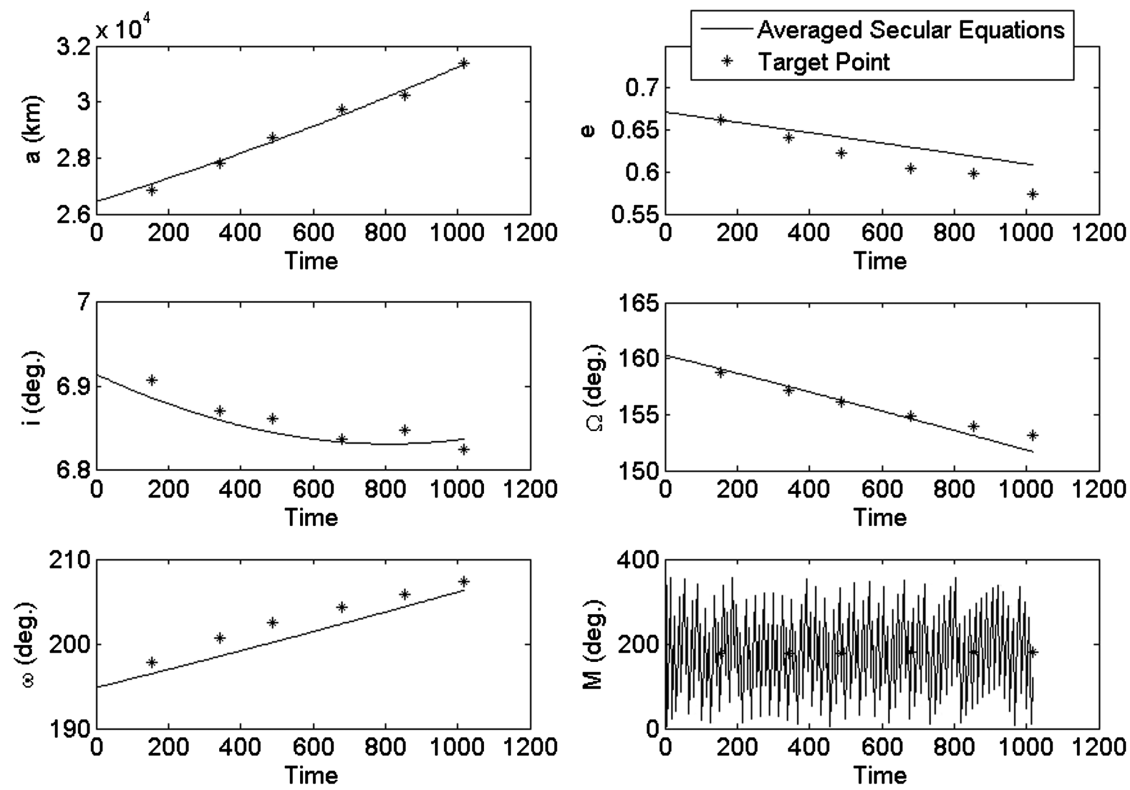

Fig. 7 SMART-1 targeting example: trajectory calculated by least-squares method. 


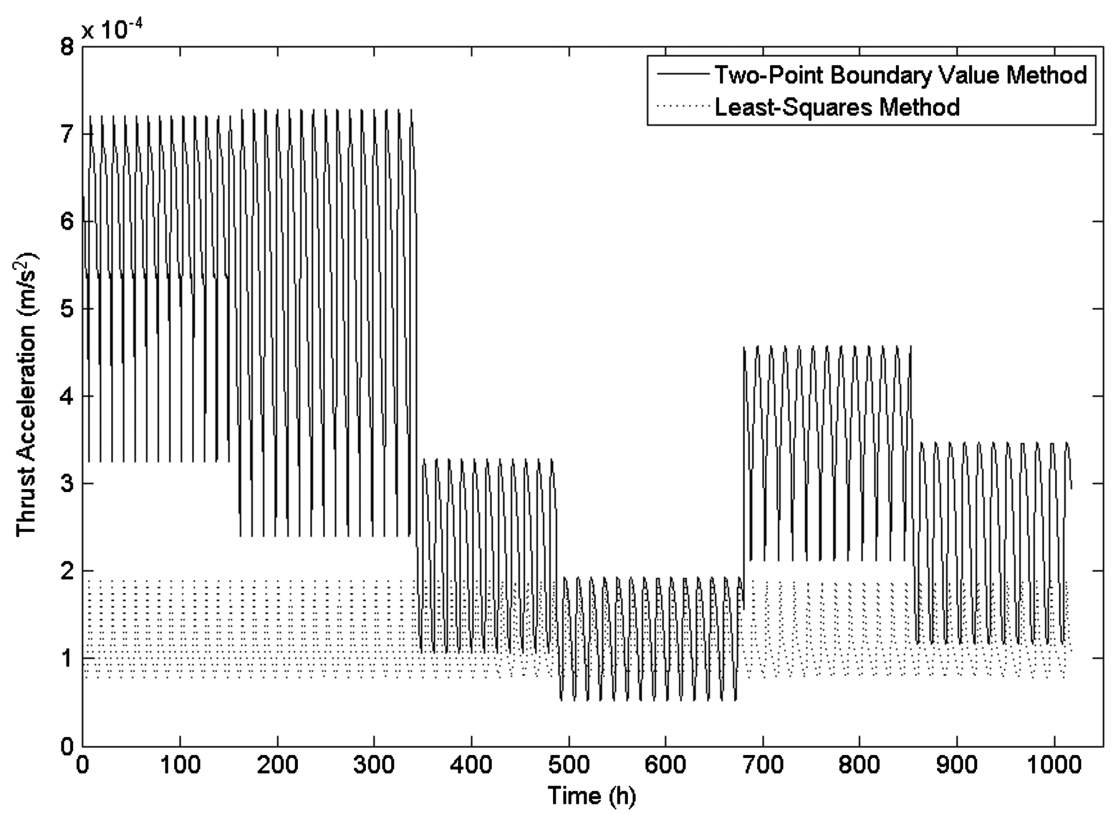

Fig. 8 SMART-1 targeting example: thrust acceleration magnitude $\left(\sqrt{F_{R}^{2}+F_{W}^{2}+F_{S}^{2}}\right)$ of control laws calculated by both methods.
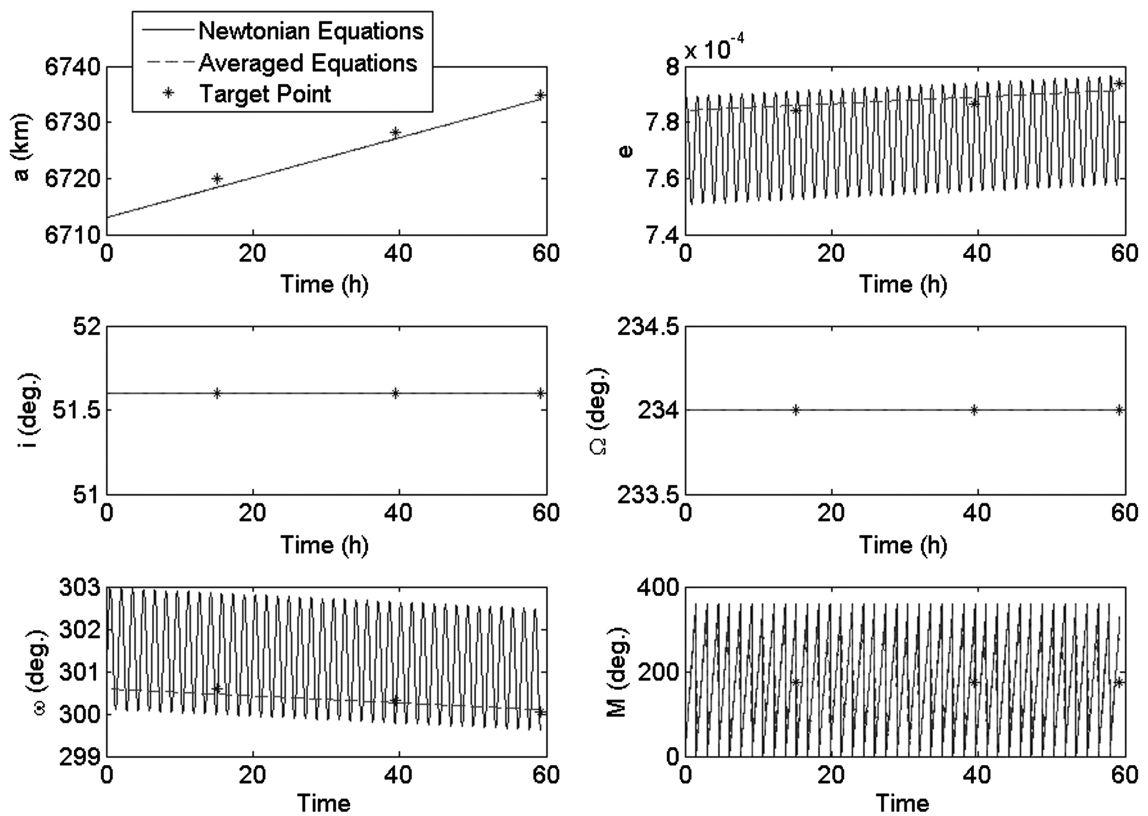

Fig. 9 Orbital element trajectory calculated by least-squares method for SSA targeting example.

$$
\begin{aligned}
\delta \boldsymbol{\alpha} & =\left[-\boldsymbol{\alpha}_{0}^{T}\left(Q-\frac{1}{2} e Z\right)-\sum_{i=1}^{N}\left[\mathbf{x}\left(\boldsymbol{\alpha}_{0}, t_{i}\right)-\mathbf{x}_{i}\right] w \Psi_{i}\right]\left[Q-\frac{1}{2} e Z\right. \\
& \left.+\sum_{i=1}^{N} \Psi_{i}^{T} w \Psi_{i}\right]^{-1}
\end{aligned}
$$

Figures $\underline{6}$ and 7 show an example of this method applied to the same targeting problem as in Table 3 . Beginning from a fixed initial state and with zero initial thrust, the averaged secular equations were integrated and Eq. (60) was used to iteratively calculate the 14 thrust Fourier coefficients. The trajectory shown is the result after ten iterations of this least-squares targeting method, when the algorithm was terminated because the coefficients changed by a maximum of $0.1 \%$ between iterations.

The velocity increment for this transfer is $410.3 \mathrm{~m} / \mathrm{s}$, less than half the value calculated by the previous approach. As shown in Fig. 8 , the overall magnitude of the thrust calculated by the least-squares method is less than that of the two-point boundary value method for most of the simulation time. Because the least-squares algorithm was not required to rigidly satisfy all the targeting criteria, it was able to find a significantly lower-cost solution to the problem. Applications where these average solutions may be useful, and where precise targeting may be eschewed to find a single control law for the entire orbit transfer, are discussed in Sec. IV.

The $\Delta V$ result from the least-squares approach is comparable to the actual SMART-1 spacecraft over the same interval. From launch through the final target state of this simulation, SMART-1 had a $\Delta V$ of $665 \mathrm{~m} / \mathrm{s}$ over 72 days and 946 hours of thrust time. . This simulation does not include the first 30 days of the mission, during which the electric propulsion system was used discontinuously, but assumes continuous thrust over the remaining 42 days (1017 hours) without coast arcs.

\footnotetext{
${ }^{\S}$ Additional data available at http://sci.esa.int/science-e/www/object/ index.cfm?fobjectid=34361 [retrieved Dec. 2010$]$.
} 
Table 4 SMART-1 targeting example: mean difference between calculated average state and target state

\begin{tabular}{lcccccc}
\hline \hline & $a, \mathrm{~km}$ & $e$ & $i, \mathrm{deg}$ & $\Omega, \mathrm{deg}$ & $\omega, \mathrm{deg}$ & $M, \mathrm{deg}$ \\
\hline 2PBVP method & $-0.2135 \mathrm{e}-3$ & $3.0370 \mathrm{e}-9$ & $-1.5588 \mathrm{e}-007$ & $-5.8031 \mathrm{e}-007$ & $8.5762 \mathrm{e}-007$ & $3.4883 \mathrm{e}-004$ \\
Least-Squares method & 31.4609 & 0.0183 & -0.0094 & -0.3208 & -1.6875 & -56.4976 \\
\hline \hline
\end{tabular}

Both the two-point boundary value method and the least-squares method are able to calculate trajectories that closely approach each of the target states. Table 4 shows the mean difference between the true and calculated average value of each orbital element over the six targets. The two-point boundary value method is very accurate, but requires a different control law for each segment of the trajectory and includes discontinuities. The least-squares method is less accurate, but uses a single control law. The relative accuracy of each variable in the least-squares method can be adjusted through the weighting parameter $w$ in Eq. (57). In this solution, mean anomaly was assigned a weighting value lower than the other orbital elements.

The simulation using the two-point boundary value method required $6.2 \mathrm{~min}$ of computational time on a personal laptop

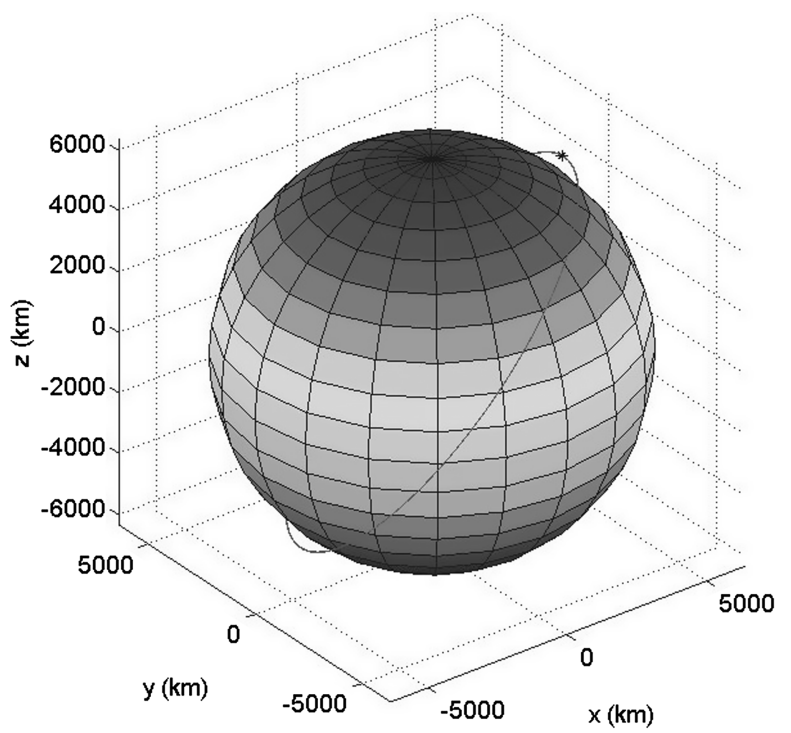

Fig. 10 3-D trajectory calculated by least-squares method for SSA targeting example. computer. The simulation using the least-squares method required 13.2 min of computational time.

\section{Applications}

Trajectory analysis using the reduced Fourier coefficients has several potential fields of application, including mission design and SSA. The different methods described above for solving orbital targeting problems have different strengths and weaknesses that make them appropriate, respectively, for these different applications.

The averaged secular equations can be used in low-thrust mission design to evaluate the control laws required for desired orbital trajectories. The solutions obtained from these equations are not, in themselves, optimal, but they satisfy the proposed targeting problems. Thus, they could serve as useful initial estimates for other optimization methods.

Using the averaged equations, mission planners can efficiently estimate the control laws for a large number of potential orbital paths, to compare the fuel costs and other trajectory characteristics. Once a baseline mission profile has been selected, these equations can be used to quickly determine the feasibility and cost of proposed deviations from the selected path.

Generally, the two-point boundary value targeting method is best suited for mission design applications. The precise agreement of the averaged trajectory with the target states is desirable, and discontinuities in the true trajectory may be inconsequential in the early mission planning stages, particularly if the solutions will later be used to initialize other optimization methods. When the target states represent flexible objectives, rather than strict targets, the segmented solutions may help mission designers to shift the target states to lower-cost alternatives. The two-point boundary value method also converges fastest and does not require the user to define a weighting matrix, which can be subjective.

SSA problems can also be solved using the averaged secular equations. Given a few discrete observations of a suspected lowthrust spacecraft, these equations can reconstruct its orbital path and identify the fundamental characteristics of the control law used. Thus

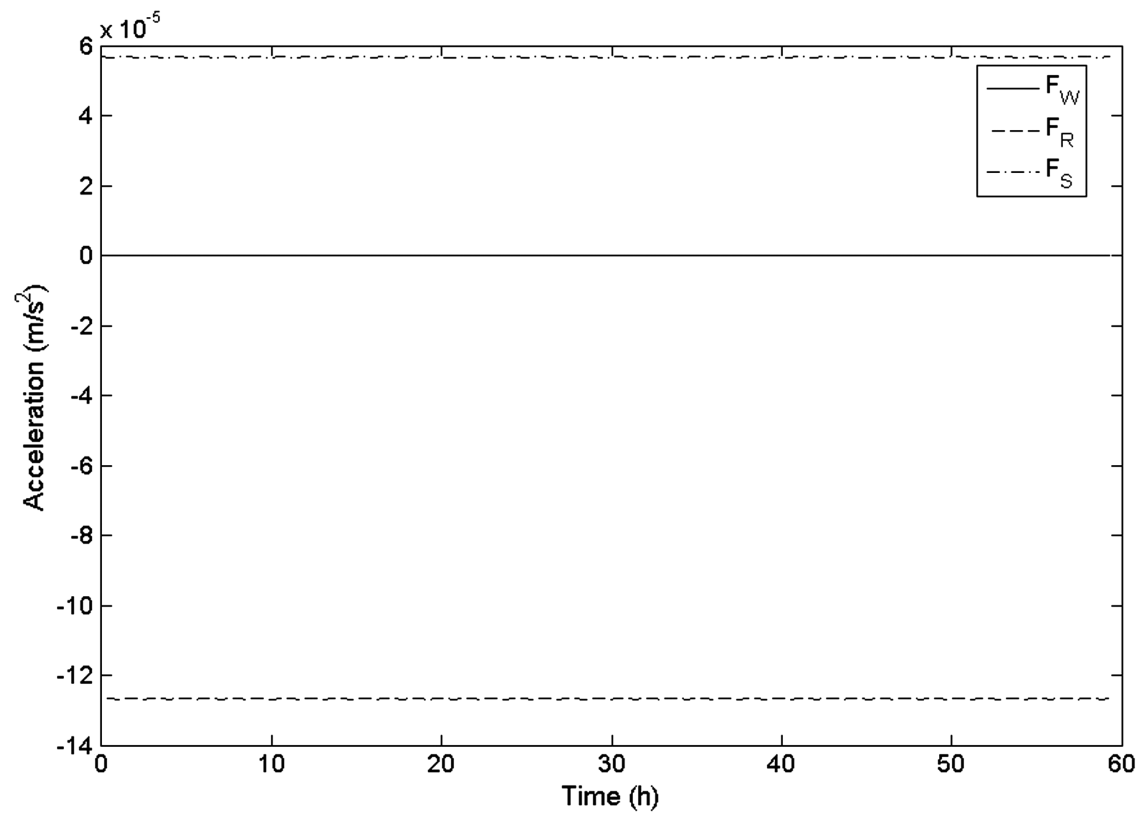

Fig. 11 Thrust acceleration magnitude calculated by least-squares methods. 
Table 5 Target states for SSA example

\begin{tabular}{lccccccc}
\hline \hline & Time, h & $a, \mathrm{~km}$ & $e$ & $i, \mathrm{deg}$ & $\Omega, \mathrm{deg}$ & $\omega, \mathrm{deg}$ & $M, \mathrm{deg}$ \\
\hline Initial State & 0 & 6713.0 & $0.7842 \mathrm{e}-3$ & 51.6 & 234.0 & 300.6 & 0 \\
Target 1 & 15.2049 & 6720.0 & $0.7841 \mathrm{e}-3$ & 51.6 & 234.0 & 300.6 & 0.0974 \\
Target 2 & 39.5328 & 6728.2 & $0.7867 \mathrm{e}-3$ & 51.6 & 234.0 & 300.3 & 0.2865 \\
Target 3 & 59.2991 & 6734.9 & $0.7936 \mathrm{e}-3$ & 51.6 & 234.0 & 300.0 & 0.8480 \\
\hline \hline
\end{tabular}

Table 6 Mean difference between calculated average state and target state for SSA example

\begin{tabular}{ccccccc}
\hline \hline & $a, \mathrm{~km}$ & $e$ & $i, \mathrm{deg}$ & $\Omega, \mathrm{deg}$ & $\omega, \mathrm{deg}$ & $M, \mathrm{deg}$ \\
\hline LSQ & -1.1257 & $6.8697 \mathrm{e}-007$ & $-3.6947 \mathrm{e}-008$ & 0.0001 & -0.0345 & -16.5010 \\
\hline \hline
\end{tabular}

observers could estimate the amount of fuel remaining and extrapolate to predict the future trajectory.

The least-squares targeting method is generally most appropriate for SSA problems. In these cases, precise agreement between the calculated and actual trajectory may be less important than simply finding a reasonable estimate for the thrust profile and fuel consumption. The SMART-1 example in Sec. III.B illustrates the process of reconstructing a trajectory from a few known states using this method. Another example of an SSA problem is shown in Table 5 and Figs. 9-11.

Table $\underline{5}$ shows a set of four states at which a hypothetical spacecraft has been observed over six days. Analysts need to quickly characterize the spacecraft's propulsion system and control law and determine its future path.

Figures 9-11 show the results of the least-squares targeting in the averaged equations through this set of states, using the 14 critical control coefficients. The targeting algorithm was terminated after five iterations, when the coefficients changed by less than $0.1 \%$ between iterations. The mean difference between the average trajectory and the target states is shown in Table 6 . In this simulation, the mean anomaly was given a low weighting value to improve the accuracy of the other orbital elements. This simulation required 2.9 min of computational time on a personal laptop computer.

The maximum total thrust acceleration was about $1.39 \mathrm{e}-4 \mathrm{~m} / \mathrm{s}^{2}$, which would require a thrust of about $69.3 \mathrm{mN}$ for a $500 \mathrm{~kg}$ spacecraft. This is well within the thrust capability of existing electric propulsion systems, such as the NASA Solar Electric Propulsion Technology Application Readiness ion thruster used on the Deep Space 1 and Dawn missions [27].

As low-thrust propulsion technology becomes increasingly popular, SSA for low-thrust spacecraft may become an area of increasing interest. Operators are more frequently using low-thrust propulsion to place satellites in orbit, creating more opportunities for collisions and radio frequency interference as these spacecraft travel slowly through altitude ranges [28]. The averaged secular equations could provide analysts with a valuable tool for quickly and accurately assessing unknown low-thrust objects.

\section{Conclusions}

The averaged secular Gauss equations were used to solve orbital targeting and optimal control problems. Two methods were developed for calculating averaged trajectories for multi-orbit transfers through a finite number of orbital states. Both methods can efficiently and accurately solve orbital targeting problems with a series of target states. The two-point boundary value method generally results in closer agreement between the average trajectory and the target states, however, it requires implementation of a new thrust vector for each interval. The calculated trajectory may have discontinuities due to short-period offsets of the true trajectory from the averaged, so this method may not be suitable for applications that require precise targeting. The least-squares method generally calculates control laws with lower dynamic fidelity, but provides a single control law for the entire, continuous trajectory.

\section{Appendix}

A simplified expression for the minimum energy cost function, Eq. (40), can be found using the Fourier coefficients given by Eqs. (31-33). The Fourier series for each component of the thrust acceleration is truncated to the key terms that appear in the average secular equations

$$
F_{R}=\alpha_{0}^{R}+\alpha_{1}^{R} \cos E+\beta_{1}^{R} \sin E+\beta_{2}^{R} \sin 2 E
$$

$$
F_{W}=\alpha_{0}^{W}+\alpha_{1}^{W} \cos E+\beta_{1}^{W} \sin E+\alpha_{2}^{W} \cos 2 E+\beta_{2}^{W} \sin 2 E
$$

$$
F_{S}=\alpha_{0}^{S}+\alpha_{1}^{S} \cos E+\beta_{1}^{S} \sin E+\alpha_{2}^{S} \cos 2 E+\beta_{2}^{S} \sin 2 E
$$

The orthogonality conditions lead to a simplified expression for Eq. (2)

$$
\begin{gathered}
a_{0}=\frac{1}{2 \pi} \int_{0}^{2 \pi} F_{R}^{2}+F_{W}^{2}+F_{S}^{2} \mathrm{~d} E \\
=\left(\alpha_{0}^{R}\right)^{2}+\frac{1}{2}\left[\left(\alpha_{1}^{R}\right)^{2}+\left(\beta_{1}^{R}\right)^{2}+\left(\beta_{2}^{R}\right)^{2}\right]+\left(\alpha_{0}^{W}\right)^{2}+\frac{1}{2}\left[\left(\alpha_{1}^{W}\right)^{2}\right. \\
\left.+\left(\alpha_{2}^{W}\right)^{2}+\left(\beta_{1}^{W}\right)^{2}+\left(\beta_{2}^{W}\right)^{2}\right]+\left(\alpha_{0}^{S}\right)^{2}+\frac{1}{2}\left[\left(\alpha_{1}^{S}\right)^{2}+\left(\alpha_{2}^{S}\right)^{2}\right. \\
\left.+\left(\beta_{1}^{S}\right)^{2}+\left(\beta_{2}^{S}\right)^{2}\right]=\frac{1}{2}\left(\boldsymbol{\alpha} \cdot \boldsymbol{\alpha}+\left(\alpha_{0}^{R}\right)^{2}+\left(\alpha_{0}^{W}\right)^{2}+\left(\alpha_{0}^{S}\right)^{2}\right)
\end{gathered}
$$

To simplify Eq. (33), note that the square of each force vector component is a sum of products of two elements of $\alpha$ and two sines or cosines of $i E$, where $i=0,1$, or 2 . For clarity, the expansion is shown for only one directional component,

$$
\begin{aligned}
F_{W}^{2} & =\left(\alpha_{0}^{W}\right)^{2}+\left(\alpha_{1}^{W}\right)^{2} \cos ^{2} E+\left(\alpha_{2}^{W}\right)^{2} \cos ^{2} 2 E+\left(\beta_{1}^{W}\right)^{2} \sin ^{2} E \\
& +\left(\beta_{2}^{W}\right)^{2} \sin ^{2} 2 E+2 \alpha_{0}^{W} \alpha_{1}^{W} \cos E+2 \alpha_{0}^{W} \alpha_{2}^{W} \cos 2 E \\
& +2 \alpha_{0}^{W} \beta_{1}^{W} \sin E+2 \alpha_{0}^{W} \beta_{2}^{W} \sin 2 E+2 \alpha_{1}^{W} \alpha_{2}^{W} \cos E \cos 2 E \\
& +2 \alpha_{1}^{W} \beta_{1}^{W} \cos E \sin E+2 \alpha_{1}^{W} \beta_{2}^{W} \cos E \sin 2 E \\
& +2 \alpha_{2}^{W} \beta_{1}^{W} \cos 2 E \sin E+2 \alpha_{2}^{W} \beta_{2}^{W} \cos 2 E \sin 2 E \\
& +2 \beta_{1}^{W} \beta_{2}^{W} \sin E \sin 2 E
\end{aligned}
$$

Using trigonometric identities, each product of sines and cosines of $i E$ can be represented as a sum of sines and cosines of $j E$, where $j=0,1,2,3$, or 4 . 


$$
\begin{aligned}
F_{W}^{2} & =\left(\alpha_{0}^{W}\right)^{2}+\frac{1}{2}\left(\alpha_{1}^{W}\right)^{2}(1+\cos 2 E)+\frac{1}{2}\left(\alpha_{2}^{W}\right)^{2}(1+\cos 4 E) \\
& +\frac{1}{2}\left(\beta_{1}^{W}\right)^{2}(1-\cos 2 E)+\frac{1}{2}\left(\beta_{2}^{W}\right)^{2}(1-\cos 4 E) \\
& +2 \alpha_{0}^{W} \alpha_{1}^{W} \cos E+2 \alpha_{0}^{W} \alpha_{2}^{W} \cos 2 E+2 \alpha_{0}^{W} \beta_{1}^{W} \sin E \\
& +2 \alpha_{0}^{W} \beta_{2}^{W} \sin 2 E+\alpha_{1}^{W} \alpha_{2}^{W}(\cos E+\cos 3 E)+\alpha_{1}^{W} \beta_{1}^{W} \sin 2 E \\
& +\alpha_{1}^{W} \beta_{2}^{W}(\sin 3 E+\sin E)+\alpha_{2}^{W} \beta_{1}^{W}(\sin 3 E-\sin E) \\
& +\alpha_{2}^{W} \beta_{2}^{W} \sin 4 E+\beta_{1}^{W} \beta_{2}^{W}(\cos E-\cos 3 E)
\end{aligned}
$$

Thus the square of each force vector component is a finite sum

$$
F_{W}^{2}=\sum_{j=0}^{4} A_{j}^{W} \cos j E+B_{j}^{W} \sin j E
$$

where

$$
\begin{aligned}
& A_{0}^{W}=\left(\alpha_{0}^{W}\right)^{2}+\frac{1}{2}\left(\alpha_{1}^{W}\right)^{2}+\frac{1}{2}\left(\alpha_{2}^{W}\right)^{2}+\frac{1}{2}\left(\beta_{1}^{W}\right)^{2}+\frac{1}{2}\left(\beta_{2}^{W}\right)^{2}, \\
& A_{1}^{W}=2 \alpha_{0}^{W} \alpha_{1}^{W}+\alpha_{1}^{W} \alpha_{2}^{W}+\beta_{1}^{W} \beta_{2}^{W}, \\
& A_{2}^{W}=\frac{1}{2}\left(\alpha_{1}^{W}\right)^{2}-\frac{1}{2}\left(\beta_{1}^{W}\right)^{2}+2 \alpha_{0}^{W} \alpha_{2}^{W}, \quad A_{3}^{W}=\alpha_{1}^{W} \alpha_{2}^{W}-\beta_{1}^{W} \beta_{2}^{W}, \\
& A_{4}^{W}=\frac{1}{2}\left(\alpha_{2}^{W}\right)^{2}-\frac{1}{2}\left(\beta_{2}^{W}\right)^{2}, \quad B_{1}^{W}=2 \alpha_{0}^{W} \beta_{1}^{W}+\alpha_{1}^{W} \beta_{2}^{W}-\alpha_{2}^{W} \beta_{1}^{W}, \\
& B_{2}^{W}=2 \alpha_{0}^{W} \beta_{2}^{W}+\alpha_{1}^{W} \beta_{1}^{W}, \quad B_{3}^{W}=\alpha_{1}^{W} \beta_{2}^{W}+\alpha_{2}^{W} \beta_{1}^{W}, \\
& B_{4}^{W}=\alpha_{2}^{W} \beta_{2}^{W}
\end{aligned}
$$

This sum, and the analogous sums for the other two directional components, are substituted for $f(\mathbf{F})$ in Eq. (33). Then the orthogonality conditions are applied again to find a simplified expression for $a_{1}$

$$
\begin{aligned}
a_{1} & =\frac{1}{2}\left(2 \alpha_{0}^{R} \alpha_{1}^{R}+\alpha_{1}^{R} \alpha_{2}^{R}+2 \alpha_{0}^{S} \alpha_{1}^{S}+\alpha_{1}^{S} \alpha_{2}^{S}+\beta_{1}^{S} \beta_{2}^{S}+2 \alpha_{0}^{W} \alpha_{1}^{W}\right. \\
& \left.+\alpha_{1}^{W} \alpha_{2}^{W}+\beta_{1}^{W} \beta_{2}^{W}\right)
\end{aligned}
$$

\section{Acknowledgments}

This material is based upon work supported under a National Science Foundation Graduate Research Fellowship. Any opinions, findings, conclusions, or recommendations expressed in this publication are those of the authors and do not necessarily reflect the views of the National Science Foundation.

Daniel J. Scheeres acknowledges support from U.S. Air Force Office of Scientific Research grant FA9550-08-1-0460.

\section{References}

[1] Forbes, G. F., "The Trajectory of a Powered Rocket in Space," Journal of the British Interplanetary Society, Vol. 9, No. 2, 1950, pp. 75-79.

[2] Tsu, T. C., "Interplanetary Travel by Solar Sail," Journal of the American Rocket Society, Vol. 29, 1959, pp. 422-427.

[3] Pinkham, G., "Reference Solution for Low Thrust Trajectories," Journal of the American Rocket Society, Vol. 32, No. 5, 1962, pp. 775776.

[4] Petropoulos, A. E., and Longuski, J. M., "Shape-Based Algorithm for Automated Design of Low-Thrust, Gravity-Assist Trajectories," Journal of Spacecraft and Rockets, Vol. 41, No. 5, 2004, pp. 787-796. doi:10.2514/1.13095

[5] Tsien, H. S., "Take-Off from Satellite Orbit," Journal of the American Rocket Society, Vol. 23, No. 4, 1953, pp. 233-236.

[6] Battin, R. H., An Introduction to the Mathematics and Methods of Astrodynamics, AIAA Education Series, AIAA, New York, 1959, pp. 408-415.

[7] Prussing, J., and Coverstone-Carroll, V., "Constant Radial Thrust Acceleration Redux," Journal of Guidance, Control, and Dynamics, Vol. 21, No. 3, 1998, pp. 516-518. doi: $10.2514 / 2.7609$

[8] McInnes, C., "Orbits in a Generalized Two-Body Problem," Journal of Guidance, Control, and Dynamics, Vol. 26, No. 5, 2003, pp. 743-749. doi: $10.2514 / 2.5129$

[9] Yamakawa, H., "Optimal Radially Acclerated Interplanetary Trajectories," Journal of Spacecraft and Rockets, Vol. 43, No. 1, 2006, pp. 116-120. doi: $10.2514 / 1.13317$

[10] Topputo, F., Owis, A., and Bernelli-Zazzera, F., "Analytical Solution of Optimal Feedback Control for Radially Accelerated Orbits," Journal of Guidance, Control, and Dynamics, Vol. 31, No. 5, 2008, pp. 13521359.

doi:10.2514/1.33720

[11] Markopoulos, N., "Explicit, Near-Optimal Guidance For PowerLimited Escape from a Circular Orbit," AIAA Guidance, Navigation and Control Conference, AIAA Paper 96-3706, July 1996.

[12] Lawden, D. F., "Optimal Intermediate-Thrust Arcs in a Gravitational Field," Astronautica Acta, Vol. 8, 1962, pp. 106-123.

[13] Bishop, R. H., and Azimov, D. M., "Analytical Space Trajectories for Extremal Motion with Low-Thrust Exhaust-Modulated Propulsion," Journal of Spacecraft and Rockets, Vol. 38, No. 6, 2001, pp. 897-903. doi: $10.2514 / 2.3761$

[14] Marec, J. P., and Vinh, N. X., "Optimal Low-Thrust, Limited Power Transfers Between Arbitrary Elliptical Orbits," Acta Astronautica, Vol. 4, Nos. 5-6, 1977, pp. 511-540. doi:10.1016/0094-5765(77)90106-0

[15] Kluever, C. A., and Oleson, S. R., "Direct Approach for Computing Near-Optimal Low-Thrust Earth-Orbit Transfers," Journal of Spacecraft and Rockets, Vol. 35, No. 4, 1998, pp. 509-515. doi: $10.2514 / 2.3360$

[16] Kluever, C. A., "Simple Guidance Scheme for Low-Thrust Orbit Transfers," Journal of Guidance, Control, and Dynamics, Vol. 21, No. 6, 1998, pp. 1015-1017. doi: $10.2514 / 2.4344$

[17] Kluever, C. A., "Low-Thrust Orbit Transfer Guidance Using an Inverse Dynamics Approach," Journal of Guidance, Control, and Dynamics, Vol. 18, No. 1, 1995, pp. 187-189. doi: $10.2514 / 3.56676$

[18] Kluever, C. A., and O'Shaughnessy, D. J., "Trajectory-Tracking Guidance Law for Low-Thrust Earth-Orbit Transfers," Journal of Guidance, Control, and Dynamics, Vol. 23, No. 4, 2000, pp. 754756. doi:10.2514/2.4597

[19] Ilgen, M. R., "Low Thrust OTV Guidance Using Lyapunov Optimal Feedback Control Techniques," AIAA/AAS Astrodynamics Specialist Conference, American Astronautical Society Paper 93-680, August 1993.

[20] Gurfil, P., "Nonlinear Feedback Control of Low-Thrust Orbital Transfer in a Central Graviational Field," Acta Astronautica, Vol. 60, No. 8-9, 2007, pp. 631-648.

doi:10.1016/j.actaastro.2006.10.001

[21] Petropoulos, A. E., "Some Analytic Integrals of the Averaged Variational Equations for a Thrusting Spacecraft," Interplanetary Network Progress Report 42-150, Jet Propulsion Lab., California Inst. of Technology, Pasadena, CA, Aug. 2002, pp. 1-29.

[22] Edelbaum, T. N., "Optimum Power-Limited Orbit Transfer in Strong Gravity Fields," AIAA Journal, Vol. 3, No. 5, May 1965, pp. 921-925. doi: $10.2514 / 3.3016$

[23] Gao, Y., "Near-Optimal Very Low-Thrust Earth-Orbit Transfers and Guidance Schemes," Journal of Guidance, Control, and Dynamics, Vol. 30, No. 2, 2007, pp. 529-539. doi: $10.2514 / 1.24836$

[24] Gao, Y., "Low-Thrust Nonlinear Guidance by Tracking Mean Orbital Elements," Journal of Guidance, Control, and Dynamics, Vol. 31, No. 4, 2008, pp. 1103-1110. doi: $10.2514 / 1.31256$

[25] Hudson, J., and Scheeres, D., "Reduction of Low Thrust Continuous Controls for Trajectory Dynamics," Journal of Guidance, Control, and Dynamics, Vol. 32, No. 3, 2009, pp. 780-787. doi:10.2514/1.40619

[26] Gustafson, E., and Scheeres, D., "Optimal Timing of Control-Law Updates for Unstable Systems with Continuous Control," Journal of Guidance, Control, and Dynamics, Vol. 32, No. 3, 2009, pp. 878-887. doi: $10.2514 / 1.38570$

[27] Polk, J., Kakuda, R., Anderson, J., Brophy, J., Rawlin, V., Patterson, M., Sovey, J., and Hamley, J., "Validation of the NSTAR Ion Propulsion System on the Deep Space One Mission: Overview and Initial Results," AIAA/ASME/SAE/ASEE Joint Propulsion Conference, AIAA Paper 992274, June 1999.

[28] Ailor, W., "Space Traffic Control: A View of the Future," Space policy, Vol. 18, No. 2, 2002, pp. 99-105. doi:10.1016/S0265-9646(02)00002-4 